Tropical Journal of Pharmaceutical Research November 2021; 20 (11): 2433-2441

ISSN: $1596-5996$ (print); 1596-9827 (electronic) (C) Pharmacotherapy Group, Faculty of Pharmacy, University of Benin, Benin City, 300001 Nigeria.

\title{
Prediction of steady-state plasma concentrations of olanzapine in Chinese Han in patients based on a retrospective population pharmacokinetic model
}

\author{
Xiaoyue Wang ${ }^{1}$, Yong Han², Hong Zhou'², Bin $\mathrm{Cao}^{3}$, Miaomiao Zhu ${ }^{1}$, Chunfang \\ Liu $^{1}$, Chao Gao', Howard L McLeod ${ }^{4}$, Maosheng Fang ${ }^{1 *}$ \\ ${ }^{1}$ Department of Pharmacy, Wuhan Mental Health Center, ${ }^{2}$ Department of Pharmacy, Union Hospital, Tongji Medical College, \\ Huazhong University of Science and Technology, Wuhan, ${ }^{3}$ Department of Information, Wuhan Mental Health Center, Wuhan, \\ China, ${ }^{4}$ Taneja College of Pharmacy and the Geriatric Oncology Consortium, University of South Florida, Tampa FL 33618, \\ USA
}

*For correspondence: Email: fangmaosheng2006@163.com; Tel: +86-027-85869112

Sent for review: 7 July 2021

Revised accepted: 19 October 2021

\begin{abstract}
Purpose: To develop robust methods of establishing a population pharmacokinetics (Pop-PK) model of olanzapine, using existing hospital in-patient information, in order to predict the steady-state plasma concentration of olanzapine tablets in Chinese Han inpatients, thus providing guidance for individualized therapy for mental disorders.

Methods: A retrospective study analyzing and predicting the steady-state plasma olanzapine concentration was performed using nonlinear mixed-effect modeling (Phoenix® NLME8). The effects of ten potential covariates, including age, gender, Body Mass Index, fasting lipid, family history, alcohol and smoking status in 107 Chinese Han patients with steady-state plasma olanzapine concentration were collected from the hospital information system (HIS) in Wuhan Mental Health Center from Feb 2017 to Jul 2019.

Results: The final model was validated using bootstrap and visual predictive check (VPC) and was found to fit the one-compartment mixed error model. Smoking status was found to be the only factor affecting olanzapine tablets clearance. The standard Pop-PK parameters apparent volume of distribution (VL/F) and clearance (CL/F) were $223 L$ and $12.4 L \cdot h-1$, respectively.

Conclusion: The Pop-PK model for olanzapine established with the data from HIS is effective in predicting the plasma olanzapine tablets concentration of individual Chinese in-patients. This Pop-PK model approach can now be adapted to optimize other antipsychotic drugs.
\end{abstract}

Keywords: Population pharmacokinetics, Plasma, Steady-state, Olanzapine, Retrospective model, Chinese Han

\begin{abstract}
This is an Open Access article that uses a funding model which does not charge readers or their institutions for access and distributed under the terms of the Creative Commons Attribution License (http://creativecommons.org/licenses/by/4.0) and the Budapest Open Access Initiative (http://www.budapestopenaccessinitiative.org/read), which permit unrestricted use, distribution, and reproduction in any medium, provided the original work is properly credited.

Tropical Journal of Pharmaceutical Research is indexed by Science Citation Index (SciSearch), Scopus, International Pharmaceutical Abstract, Chemical Abstracts, Embase, Index Copernicus, EBSCO, African Index Medicus, JournalSeek, Journal Citation Reports/Science Edition, Directory of Open Access Journals (DOAJ), African Journal Online, Bioline International, Open-J-Gate and Pharmacy Abstracts
\end{abstract}

\section{INTRODUCTION}

A mental disorder is a behavioral or mental pattern that is caused by the dysfunctioning of the central nervous system, and it results in significant distress or impairment of personal functioning [1]. It is estimated that there are approximately 1 billion people with mental disorders worldwide, and more than 240 million of these patients are in China [2]. Olanzapine is a 
novel therapy, and the second most commonly used atypical antipsychotic drug (13.1\% of prescriptions) for the treatment of mental disorders in China [3]. Olanzapine has demonstrated efficacy in ameliorating both the negative and positive symptoms of schizophrenia. Patients receiving olanzapine treatment have shown fewer extrapyramidal adverse effects than those receiving firstgeneration antipsychotics (FGA) [4]. Unfortunately, olanzapine can often result in assorted adverse drug reactions (ADRs), such as cardiovascular disease, lipid metabolism disorder, diabetes mellitus, motor side effects, and weight gain, and the severity of these ADRs is dose-dependent [5]. The clinical efficacy of olanzapine was previously shown to be influenced by its plasma concentration [6].

Patients with slower-than-average drug clearance have a higher risk of developing adverse effects. However, classical pharmacokinetics (PK) is insufficient for explaining the correlation between individual variations and the clinical response of patients. Close monitoring of olanzapine concentration is therefore required for clinical treatment [7]. Population pharmacokinetic (Pop-PK) methodologies analyze the sources of variation through TDM data. Pop-PK model also allows the identification of potential pharmacokinetic drug interactions and variability factors that affect drug efficacy based on both rich and sparse data [8].

Previous studies showed that race, gender, body weight, age and other potential factors may affect olanzapine systemic exposure [9-12]. How these factors affect olanzapine concentration has yet to be a subject of critical study in China. In this study, a Pop-PK model for accurate prediction of plasma olanzapine concentration was built using the available data at Hospital Information Systems (HIS), and a feasibility study to examine its rapid establishment in other antipsychotic drugs was verified, so as to improve individualized treatments in mental disorder patients.

\section{METHODS}

\section{Patients}

Chinese Han in-patients who received chronic olanzapine table treatment were retrospectively identified from the HIS in Wuhan Mental Health Center from Feb. 2017 to July 2019. The inclusion criteria were: 1) Chinese Han inpatients who received chronic olanzapine tablets treatment for more than a year; 2) patients with normal liver and kidney functions; 3) patient who are $\geq 18$ years of age; 4 ) patients who have taken olanzapine tablets for over 7 days without dose adjustment; and 5) - samples with steady-state concentration. The exclusion criteria included unclear dose adherence, use of disintegrating tablets, missing information, etc. A total of 107 Chinese Han inpatients were included, as well as information on when the samples were obtained (range of days after starting olanzapine, range of times of day). This study was conducted at the Mental Health Center of Wuhan and was approved by the institutional review board at the participating site (KY2018.19) and also complied with international guidelines for human studies [13].

\section{Evaluation of specimens}

Plasma concentration of olanzapine was measured using liquid chromatography tandem mass spectrometry (LC/MS/MS) [14], and liquid chromatography was performed using Agilent XDB-C18 $(4.6 \mathrm{~mm} \times 50 \mathrm{~mm}, 1.8 \mu \mathrm{m})$ column. Methanol-water solution (ammonium $5 \mathrm{mmol} \cdot \mathrm{L}-1$ ) $(85: 15, V / V)$ was used as the mobile phase, and the detection wavelength was $0.5 \mathrm{~mL} \cdot \mathrm{min}^{-1}$. The column temperature was $35^{\circ} \mathrm{C}$, and the injection volume was $1 \mu \mathrm{L}$.

Mass spectrometry condition (MS) was performed using electrospray source ionization (ESI), multiple response monitoring mode (MRM), and positive ion scanning. Capillary voltage was set at $4000 \mathrm{~V}$, and nebulizer pressure at $344.75 \mathrm{kPa}$, with drying gas flow and dry gas temperature set at $10 \mathrm{~L}$ min- 1 and $350{ }^{\circ} \mathrm{C}$ respectively. Quantitative ion-pair analysis of olanzapine (m/z313. $2 \rightarrow \mathrm{m} / \mathrm{z} \quad 256.1) \quad$ was conducted using $22 \mathrm{eV}$ collision energy and 160 eV fragmentation, while qualitative ion-pair analysis of olanzapine (m/z313. $2 \rightarrow \mathrm{m} / \mathrm{z}$ 84.2) was conducted using $20 \mathrm{eV}$ collision energy, and $155 \mathrm{eV}$ fragmentation. Internal standard for olanzapine was D3 m/z 316.1 $\rightarrow \mathrm{m} / \mathrm{z} 256.0,22 \mathrm{eV}$ collision energy, and $160 \mathrm{eV}$ fragmentation. The electron multiplier was $300 \mathrm{eV}$, and the extraction recoveries of the samples were above $90 \%$ for olanzapine.

\section{Population pharmacokinetic analysis}

\section{Base model selection}

Ten potential covariates, such as BMI, age, gender, fasting lipid, triglycerides (TG), total cholesterol (TCHO), low-density cholesterol (LDL), high-density cholesterol (HDL)), family history, dose, drinking habit (a history of habitual drinking regularly for over 6 months of more than 
14 units of alcohol per week), smoking status ( $\geq 5$ cigarettes per day), and concomitant medications were evaluated. Continuous covariates (BMI, age and fasting lipids) were incorporated with a power function after normalizing the median value. The plasma concentration-time data of olanzapine was analyzed using the non-linear mixed-effects model in Phoenix® NLME8 (Certara USA, Inc., 103 Carnegie Center, Suite 300, Princeton, NJ 08540 USA) and the FirstOrder Conditional Estimation Extended Least Squares method (FOCE ELS). The data set was obtained from the fixed blood concentration monitoring in the hospital where the fixed monitoring was carried out $10.5 \mathrm{~h}$ after administration. Hence, a one-compartment model with first-order absorption and elimination was used as the base model to investigate the combined error, as well as the proportional and additive error models. The best model was selected based on the changes in object function value (OFV), goodness-of-fit and parameter rationality.

\section{Initial parameter estimate}

The values of the parameters were obtained by fitting the data set, with Naïve pooled data set as the initial values for the base model.

\section{Parameter model}

An exponential model was chosen as the interindividual variability model, and the relationship between a parameter $(P)$ and its variance is expressed, as shown in Eq 1.

$P=\operatorname{tvP} \times \exp (n P)$

where $P$ is the main pharmacokinetic parameters, tvP is the typical value of $P$ for the population, and $\mathrm{nP}$ denotes the inter-individual variability of pharmacokinetic parameters.

\section{Covariate model selection and final model}

Categorical covariates such as gender, smoking status, and concomitant medications, were included in the model with an exponential function. Visual screening was performed before modeling, using scatterplots for continuous variables and box plots for categorical variables. Important covariates showing potential relationships with certain Pop-PK parameters were identified using visual screening. Continuous covariates (Covariate-dPdCovariate) were introduced into each parameter in a stepwise. Categorical covariates $(\exp (\mathrm{dPdCovariate} \cdot($ Covariate $=1)) \quad$ were introduced into each parameter, where
$\mathrm{dPdCovariate}$ was the fixed effect of $\mathrm{P}$ and Covariate.

\section{Covariate selection}

Stepwise forward selection-backward elimination with a likelihood-ratio test that was twice the log likelihood (-2LL) was used for estimating how well the model fits. Significance criteria for forward selection was 0.01 , and for backward elimination was 0.001 .

\section{Model validation and simulation}

A non-parametric bootstrap method $(n=500)$ was performed to assess the precision of estimated parameters. The final model parameter estimates obtained from the dataset were compared with medians and $95 \% \mathrm{Cls}$ of the bootstrap estimates. Adequacy of the final model was simultaneously evaluated using bootstrapping and visual predictive checks (VPC). A VPC was performed using 1000 dataset simulations to evaluate model performance.

\section{Prediction}

Two plasma samples at 2 given time points were retrospectively collected for the measurement of plasma concentrations. There were at least 7 half-life intervals between the first and the second plasma concentrations. The established population pharmacokinetic model of olanzapine was adopted to estimate individual pharmacokinetic parameters using the first concentration value, so as to predict the followup plasma concentration value. The accuracy of the model prediction was evaluated by comparing the real measured value with the predicted one.

\section{RESULTS}

\section{Demographic characteristics}

One hundred and seven psychiatric inpatients were included in this research. A total of 107 inpatients (age range 18 - 83 years) with 319 data points participated in this research. The gender ratio was almost equal. The median BMI of inpatients was 20.7. Smokers, drinkers and inpatients with family genetic history made up $29.9 \%, 10.3 \%$ and $15.0 \%$, respectively. The demographic characteristics of all enrolled subjects are shown in Table 1. Concomitant medications those were used in $1 \%$ or more inpatients are shown in Table 2. 
The plasma concentration data was obtained from the fixed blood concentration monitoring (10.5 $\mathrm{h}$ after administration). It was best described using one-compartment mixed error model.

\section{Base model}

The first-order absorption one-compartment linear elimination model was used in the pharmacokinetic model, and the additive, proportional and mixed types were investigated in the error model. The Naive Pooled method was used to get the initial value of each parameter for the data and analysis, and FirstOrder Conditional Estimation Extended Least Squares method (FOCE ELS) algorithm was used to fit the model. The results showed that the
Akaike information criterion (AIC) values of addition type, proportional type and mixed type were 2407, 2357 and 2354 respectively (Table 3 ). The results of Akaike information criterion (AIC) and goodness-of-fit showed that the mixed error model was the proper fit for the plasma concentration data, and this model was chosen as the Base model for subsequent Pop-PK analysis (Figure 1). The population parameter estimates of the Base model are shown in Table 4.

\section{Covariate selection and final model}

The covariant vs Eta scatter diagram or box diagram were drawn for exploratory analysis. The stepwise covariates were used to screen the covariates of the model.

Table 1: Patient demographics

\begin{tabular}{lcc}
\hline Characteristic & Value (mean $\mathbf{\text { SD}}$ ) & Median (range) \\
\hline Gender (male/female) $(\mathrm{n})$ & $52 / 55$ & \\
Smoking (yes/no) $(\mathrm{n})$ & $32 / 75$ & \\
Drinking(yes/no) $(\mathrm{n})$ & $11 / 96$ & \\
Family History(yes/no) $(\mathrm{n})$ & $16 / 91$ & $20.7(15.6-30.9)$ \\
BMI (kg/m2) & $20.65 \pm 3.45$ & $34(18-83)$ \\
Age (years) & $39.83 \pm 18.2$ & $2.21(0.51-5.08)$ \\
LDL (mmol/L) & $2.29 \pm 0.73$ & $1.19(0.44-2.22)$ \\
HDL (mmol/L) & $1.22 \pm 0.26$ & $4.44(2.46-7.95)$ \\
TCHO (mmol/L) & $4.46 \pm 0.88$ & $1.50(0.45-7.05)$ \\
TG (mmol/L) & $1.77 \pm 1.02$ & \\
\hline
\end{tabular}

Table 2: Concomitant medications used in $1 \%$ or more inpatients

\begin{tabular}{lccc}
\hline Medication & Use (\%) & Dose [mean \pm SD (median, range)] & Unit \\
\hline Shengxuebao Mixture & 3.07 & $15.04 \pm 1.54(10,30)$ & $\mathrm{mL}$ \\
Risperidone tablets & 2.95 & $1.29 \pm 0.66(0.25,3)$ & $\mathrm{mg}$ \\
Potassium chloride sustained-release & 2.42 & $0.96 \pm 0.21(0.5,2)$ & $\mathrm{g}$ \\
tablets & 2.16 & $0.36 \pm 0.10(0.2,0.8)$ & $\mathrm{mg}$ \\
Alprazolam tablets & 2.04 & $2,147.65 \pm 729.46(1000,3000)$ & $\mathrm{mg}$ \\
Vitamin C injection & 1.99 & $40.41 \pm 2.86(40,60)$ & $\mathrm{mg}$ \\
Adenosine triphosphate injection & & &
\end{tabular}

Table 3: Analysis results of three error models

\begin{tabular}{lll}
\hline Error model & Algorithm & AIC \\
\hline Add & FOCE ELS & 2407.2597 \\
Mult & FOCE ELS & 2357.2521 \\
Mixed & FOCE ELS & 2354.8343 \\
\hline
\end{tabular}

Table 4: Population parameter estimates of the Base model

\begin{tabular}{lccccc}
\hline Parameter & $\begin{array}{c}\text { Estimate } \\
\text { (Shrinkage) }\end{array}$ & $\begin{array}{c}\text { Standard } \\
\text { error }\end{array}$ & RSE & $\mathbf{2 . 5 \%} \mathbf{~ C l}$ & $\mathbf{9 7 . 5 \%} \mathbf{~ C l}$ \\
\hline tvKa & 20.4 & 6.09 & 29.9 & 8.38 & 32.3 \\
tvV & 210 & 41.3 & 19.7 & 129 & 291 \\
tvCl & 13.5 & 0.690 & 5.13 & 12.1 & 14.8 \\
Random effects & & & & & \\
w2Cl & $0.0914(11.1)$ & 0.0160 & 17.5 & 0.123 & 0.0600 \\
Residual error & & & & & \\
Proportional & 0.260 & 0.0200 & 8.11 & 0.220 & 0.310 \\
Additive & 2.96 & 1.13 & 38.3 & 0.730 & 5.19 \\
\hline
\end{tabular}




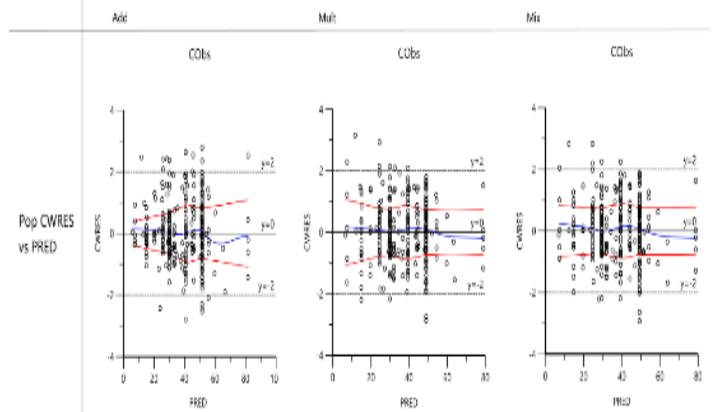

Figure 1: Pop CWRES vs. PRED plot of three error models

With -2LL as the objective function, the statistically significant differences between forward inclusion and backward elimination were $p<0.01$ and $p<0.001$, respectively. The $-2 \mathrm{LL}$ value of the basic model is 2345.029 . The final Pop-PK model for chronic olanzapine tablets treatment in Chinese Han inpatients was a onecompartment mixture model with additive error. Population parameter estimates of the final model and olanzapine clearance (smokers and nonsmokers) are shown in Table 5 and Table 6 , separately.

$$
C=\operatorname{Dose} \cdot e^{-\frac{\operatorname{tv} C L \cdot e^{d C l d I S M ~ 1 \cdot(I S M=1)} \cdot e^{\mathrm{nCl}}}{V} \cdot \mathrm{t}}
$$

Diagnostic plots of goodness-of-fit for the final model are shown in Figure 2. The fits for the observed value versus the PRED concentration (Figure $2 \mathrm{~A}$ ) and the observed value versus the IPRED concentration (Figure 2 B) were good, and also aligned on the line of identity. Both fit plots showed random distribution around zero, and did not reflect any systemic deviations. Overall, the plots indicated that the study data was sufficiently well described by the final model developed in this study.
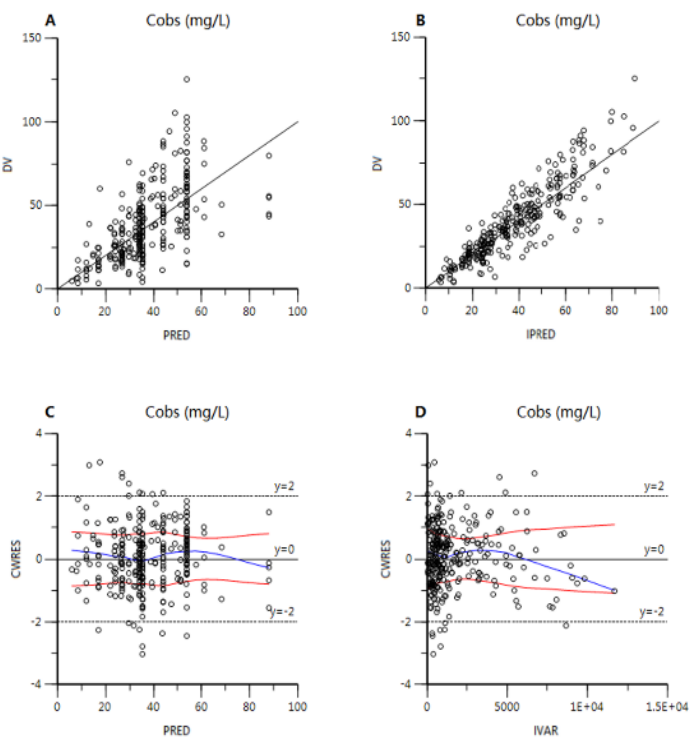

Figure 2: Goodness-of-fit plots of the final model. (A) Observed concentration vs. IPRED; (B) Observed concentration vs. PRED; (C) Conditional weighted residual error (CWRES) vs. PRED; (D) CWRES vs. time

\section{Model evaluation}

The PK parameter estimates of the final model were consistent with those of the bootstrap after 500 runs, which indicates that the model is robust and stable (Table 7 ).

Table 5: Population parameter estimates of the final model

\begin{tabular}{|c|c|c|c|c|c|}
\hline Parameter & $\begin{array}{c}\text { Estimate } \\
\text { (Shrinkage) }\end{array}$ & Standard error & RSE & $2.5 \% \mathrm{Cl}$ & $97.5 \% \mathrm{Cl}$ \\
\hline tvKa & 20.6 & 3.59 & 17.4 & 13.6 & 27.7 \\
\hline tvV & 223 & 41.3 & 18.5 & 142 & 305 \\
\hline $\operatorname{tvCl}$ & 12.4 & 0.608 & 4.92 & 11.2 & 13.6 \\
\hline dCIdISM1 & 0.319 & 0.0639 & 20.1 & 0.193 & 0.444 \\
\hline \multicolumn{6}{|l|}{ Random effects } \\
\hline (1) $2 \mathrm{Cl}$ & $0.0732(0.142)$ & 0.0136 & 18.6 & 0.0465 & 0.09 .98 \\
\hline \multicolumn{6}{|l|}{ Residual error } \\
\hline Probortional va & 0.264 & 0.0216 & 8.18 & 0.221 & 0.306 \\
\hline Additive & 2.98 & 1.17 & 391 & 0.685 & 527 \\
\hline
\end{tabular}

Table 6: Population olanzapine clearance

\begin{tabular}{lccc}
\hline Population & Mean clearance & Standard deviation & $P$-value \\
\hline Smoking status & & & $<0.001$ \\
Smokers $(n=33)$ & 17.5 & 4.42 & \\
Non-smokers $(n=74)$ & 12.7 & 2.94 & \\
\hline
\end{tabular}


Table 7: Population parameter estimates of the bootstrap validation

\begin{tabular}{llllll}
\hline Parameter & Estimate & Stderr & RSE & $\mathbf{2 . 5 \%} \mathbf{C l}$ & $\mathbf{9 7 . 5 \%} \mathbf{~ C I}$ \\
\hline tvKa & 20.3 & 0.539 & 2.66 & 18.8 & 20.7 \\
tvV & 239 & 57.3 & 24.0 & 163 & 371 \\
tvCl & 12.4 & 0.623 & 5.02 & 11.2 & 13.8 \\
dCldISM1 & 0.318 & 0.0658 & 20.7 & 0.196 & 0.444 \\
$\begin{array}{l}\text { Random effects } \\
\text { w2Cl }\end{array}$ & & & & & \\
Residual error & 0.0724 & 0.0138 & 0.190 & 0.0470 & 0.101 \\
$\begin{array}{l}\text { Proportional } \\
\text { Additive }\end{array}$ & 0.262 & & & & \\
\hline
\end{tabular}

The VPC results of the final model showed that the observed plasma concentration data mostly fitted well within the 5th-95th percentiles of the simulated data, suggesting that the final model adequately explained the observed data (Figure 2). Smoking history was used as the stratification factor for the VPC plot, and it showed that the correlation between non-smoking inpatients and smoking inpatients was appropriately described (Figure 3).
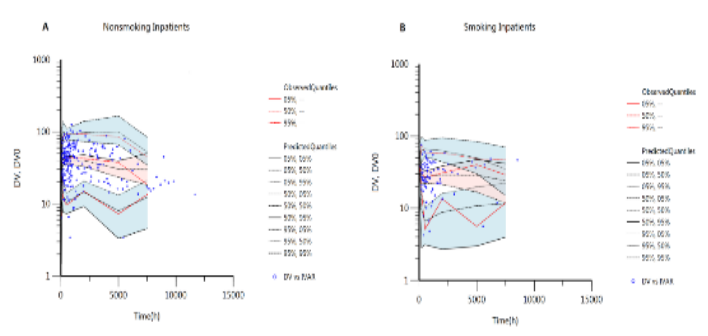

Figure 3: Correlation VPC plot for the final model. (A) non-smoking inpatients, (B) smoking inpatients

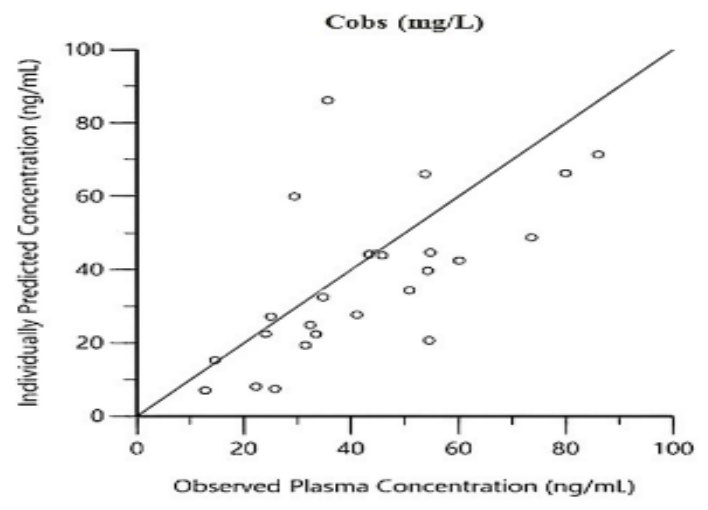

Figure 4: Relationship between observed and predicted plasma concentrations of olanzapine

\section{Concentration prediction}

Data was retrospectively collected from 25 patients for plasma concentration prediction. 95 patients were predicted to fall in the target range. The observed and predicted concentrations of olanzapine tablets were highly correlated $(r=$
0.91, $p<0.0001$ ) (Figure 4), which indicated that the prediction model had good predictability and accuracy. The distribution of the prediction errors is shown in Figure 5.

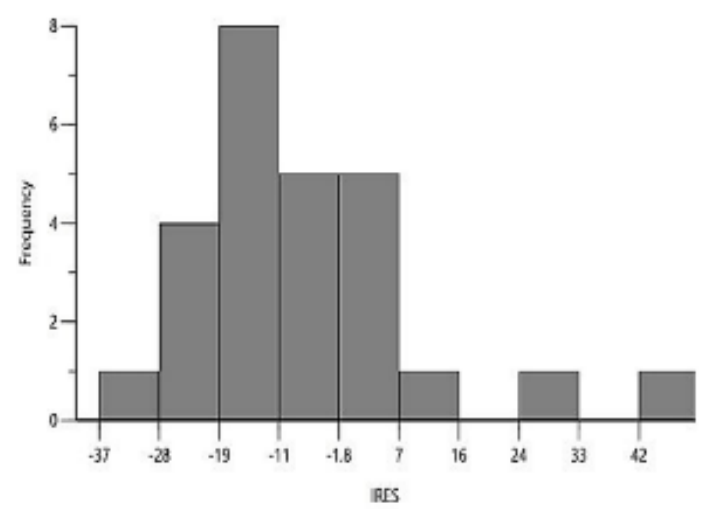

Figure 5: Distribution of prediction errors

\section{DISCUSSION}

Strategies for optimizing mental health medications are critical to providing better hope for patients and their families, and Pop-PK has proven to be a useful method for analyzing both sparse and rich data. The nonlinear mixed effect model (NLMEM) method is increasingly being used to estimate PK parameters of studies with rare sampling design, determine variability in drug exposure and detect potential pharmacokinetic drug-drug interactions, and it also plays an important role in routine TDM. Patients who receive antipsychotic medication differ with respect to treatment effects and adverse drug reactions. Thus, extensive and indepth Pop-PK researches are needed to be studied for mental disorder patients who need individualized treatment.

This Pop-PK model described the pharmacokinetics of chronic olanzapine treatment in Chinese Han inpatients. The plasma concentrations data was obtained from the fixedtime blood concentration monitoring (10.5 $\mathrm{h}$ after administration), and the information was very limited. The results of the shrinkage value of parameters for tvKa and tvV were too large to 
evaluate the inter-individual variations of $\mathrm{Ka}$ and $\mathrm{V}$. Thus, only the inter-individual variation of $\mathrm{Cl}$ was estimated. The one-compartment mixed error model provided an appropriate description of the PK characteristics of olanzapine. By fixing the absorption constant $\mathrm{Ka}$ at $0.51 \mathrm{~h}^{-1}$ as previously described [9], it was discovered that the population mean clearance and volume of distribution of olanzapine were $12.4 \mathrm{~L} \cdot \mathrm{h}^{-1}$ and $223 \mathrm{~L}$, respectively. These results demonstrate that smokers cleared olanzapine $38 \%$ faster than past/non-smokers $(p<0.001$, unpaired ttest). BMI, age, gender, family history, fasting lipids and drinking habits had no effect on olanzapine clearance. The Pop-PK model was aptly used to examine the effect of plasma concentration on olanzapine clearance, and to predict steady-state plasma olanzapine concentration of inpatients, which is consistent with previous reports [14].

Some studies have found that concomitant medications such as sertraline, Valproic Acid (VPA) and carbamazepine can affect the estimate of clearance $[6,12,15]$. Pop-PK is a useful method for identifying factors that contribute to variability in drug exposure as well as detecting potential pharmacokinetic interactions. Concomitant medications that were used in $1 \%$ or more inpatients were tested as discrete covariates, in order to determine their impact on olanzapine clearance, and also in order to discover other drugs which may affect olanzapine blood concentration. Shengxuebao mixture, risperidone tablets, potassium chloride sustained-release tablets, alprazolam tablets, vitamin $C$ injection, and adenosine triphosphate injection were found to have no significant effect on drug clearance. One possible explanation for this is that different formulations have different pharmacokinetic parameters and bioequivalence, and therefore their dose and effects on drug clearance had to be calculated separately [15]. The use of drugs which were reported to have an effect on the estimated clearance in previous studies with different formulation types did not meet the $1 \%$ required. Therefore, those drugs did not contain, and were not analyzed as comedications of olanzapine in this study. Furthermore, the schedules of medication in clinical therapy are complicated and the potential drug interactions will need to be confirmed.

In this study, $30.4 \%$ of Chinese Han in-patients who received chronic olanzapine treatment smoked during inpatient treatment. Smoking status was found to be the only variable that affected olanzapine clearance in the present study. Olanzapine clears faster in patients who smoke and this is a potential problem that physicians need to be aware of. Psychiatric patients are associated with tobacco use and prevalence rates. The rate of smoking behavior is higher in psychiatric patients, especially schizophrenics, than the general population. Some studies showed that tobacco is not associated with olanzapine metabolism in vivo $[16,17]$. A few other researches indicate a significant influence of cigarettes on the clinical outcome [16]. Psychiatric patients have more intense smoking and nicotine dependence. On the other hand, they have more difficulty with managing smoking prohibition. Some of them may refuse active treatment because of compulsory smoking cessation [10].

During the process of clinical treatment, health professionals do mandatory training to mental patients (some achieving results have been gotten with their mobilization efforts). At the same time, psychiatric inpatients are allowed to smoke outside or in designated smoking room. The research showed that the dose of olanzapine should be increased in smoking patients as a result of accelerated olanzapine clearance. Patients who quit smoking should receive a corresponding dose reduction to prevent the risk of side effects. Application of popPK provides a better way of capturing variation in an individual patient, and adapting the dosage to achieve the target blood levels.

This research examined the impact of age on the clearance of olanzapine tablets. Castberg et al [17] found that olanzapine tablets concentrations were higher in patients aged between 60 and 79 years than in patients aged between 18 and 59 years. However, another study showed that age had no significant effect on plasma olanzapine concentration [11]. In this study, age was not a significant influencing factor for olanzapine tablets clearance when the smoking status was taken into consideration. The results also showed that gender had no significant effect on olanzapine clearance, which was inconsistent with the findings of previous studies abroad $[10,11,18,19]$, but was consistent with some domestic studies [20]. A possible explanation for this discrepancy is the racial difference between the subjects used in this study (Chinese Han) and the previous studies (mostly Caucasians).

An integral Pop-PK model was established for chronic olanzapine tablets treatment in Chinese Han inpatients by assessing various parameters that may influence efficacy. Using the Bayesian method to predict individual blood concentration is useful for selecting the best dosage for each patient and ensuring optimal clinical efficacy. The findings demonstrated that prediction of plasma 
olanzapine tablets concentration using the same Pop-PK model is feasible, which is consistent with the study by Tsuboi et al [21]. Based on the population pharmacokinetic model established with the available data from HIS in hospital, it is feasible to accurately predict the steady-state plasma concentrations of olanzapine through limited blood sampling points in Chinese patients, and to implement individualized dose regimen designs.

There were several limitations in this study. Firstly, smokers and nonsmokers were identified by self-reporting and nurse evaluations without objective biological measures, which resulted in a lack of proper assessment of the magnitude of their smoking status. Secondly, some factors that were shown to impact olanzapine tablets elimination in previous studies are not commonly measured in Chinese patients. For example, the variability in olanzapine tablets clearance was reported to be associated with efficacy and the severity or duration of ADRs, and genetic factors, such as CYP1A2 polymorphism, have also been suggested to play a role in the metabolism rate of olanzapine tablets $[9,19]$. How these factors may influence olanzapine tablets clearance in the Chinese population are currently unclear and will need to be investigated. Thirdly, some studies have found that concomitant medications, such as sertraline, Valproic Acid (VPA) and carbamazepine can affect the clearance estimate $[6,12,21]$. Since the use of concomitant medications during antipsychotic treatment is often complex, the PK interactions between these medications and olanzapine tablets should be further characterized.

\section{Limitation of the study}

The sample size of this study was small, and the resulting statistical data may be insufficient to draw meaningful conclusions. Nonetheless, the findings of the present study will be confirmed by further validation with large-scale samples.

\section{CONCLUSION}

The results indicate that the Pop-PK model for olanzapine established by the data from HIS is effective and feasible in predicting the plasma olanzapine concentration of Chinese patients. Smoking status can significantly affect olanzapine clearance and the change in plasma olanzapine concentration can be reliably predicted by this Pop-PK model. Thus, the Pop-PK model established in this study provides new insights into individualized therapy of olanzapine.

\section{DECLARATIONS}

\section{Acknowledgement}

This work was supported by a project funded by the National Natural Science Foundation of China (no. 81771445), the project funded by the National Natural Science Foundation of China (no. 81673456), and the Fund Project Scientific research project of Wuhan Health and Family Planning Commission (no. WX18D12). The authors would like to thank Mr. Yong-chao Fu for all of his hard work and significant contributions towards the study.

\section{Competing interests}

No conflict of interest is associated with this article.

\section{Contribution of authors}

We declare that this work was done by the authors named in this article and all liabilities pertaining to claims relating to the content of this article will be borne by the authors. Wang Xiaoyue, Fang Mao-sheng and Howard L McLeod designed the research; Wang Xiao-yue and Han Yong performed the experiments and wrote the article; Wang Xiao-yue, Fang Mao-sheng and Han Yong analyzed the data; Cao Bin, Zhu Miaomiao, Liu Chun-fang and Gao Chao helped to collect the sample and clinical data. All authors read and approved the final manuscript.

\section{Open Access}

This is an Open Access article that uses a funding model which does not charge readers or their institutions for access and distributed under the terms of the Creative Commons Attribution License (http://creativecommons.org/licenses/by/ 4.0) and the Budapest Open Access Initiative (http://www.budapestopenaccessinitiative.org/rea d), which permit unrestricted use, distribution, and reproduction in any medium, provided the original work is properly credited.

\section{REFERENCES}

1. Stein DJ. What is a mental disorder? A perspective from cognitive-affective science. Can J Psychiatry 2013; 58(12): 656-662.

2. Huang $Y$, Wang $Y$, Wang $H$, Liu Z, Yu $X$, Yan J, Yu $Y$, Kou C, XuX, Lu J, et al. Prevalence of mental disorders in China: a cross-sectional epidemiological study. Lancet Psychiat 2019; 6(3): 211-224. 
3. Xue HB, Liu L, Zhang H, Montgomery W, Treuer $T$. Olanzapine in Chinese patients with schizophrenia or bipolar disorder: a systematic literature review. Neuropsychiatr Dis Treat 2014; 10: 841-864.

4. Lally J, MacCabe JH. Antipsychotic medication in schizophrenia: a review. Br Med Bull 2015; 114(1): 169179.

5. Law S, Gudbrandsen M, Magill N, Sweetman I, Rose D, Landau S, Flanagan RJ, David AS, Patel MX. Olanzapine and risperidone plasma concentration therapeutic drug monitoring: A feasibility study. J Psychopharmacol 2015; 29(8): 933-942.

6. Davies SJ, Mulsant BH, Flint AJ, Meyers BS, Rothschild AJ, Whyte EM, Kirshner MM, Sorisio D, Pollock BG, Bies RR. SSRI-antipsychotic combination in psychotic depression: sertraline pharmacokinetics in the presence of olanzapine, a brief report from the STOP-PD study. Hum Psychopharmacol 2016; 31(3): 252-255.

7. Hiemke C, Bergemann N, Clement HW, Conca A, Deckert J, Domschke K, Eckermann G, Egberts K, Gerlach M, Greiner C, et al. Consensus Guidelines for Therapeutic Drug Monitoring in Neuropsychopharmacology: Update 2017. Pharmacopsychiatry 2018; 51(1-02): 9-62.

8. Jing L, Liu TT, Guo Q, Chen M, Lu JJ, Lv CL. Development and comparison of population pharmacokinetic models of vancomycin in neurosurgical patients based on two different renal function markers. $J$ Clin Pharm Ther 2020; 45(1): 88-96.

9. Bigos KL, Pollock BG, Coley KC, Miller DD, Marder SR, Aravagiri M, Kirshner MA, Schneider LS, Bies RR. Sex, race, and smoking impact olanzapine exposure. J Clin Pharmacol 2008; 48(2): 157-165.

10. Dome P, Lazary J, Kalapos MP, Rihmer Z. Smoking, nicotine and neuropsychiatric disorders. Neurosci Biobehav Rev 2010; 34(3): 295-342.

11. Zullino DF, Delessert $D$, Eap CB, Preisig M, Baumann $P$. Tobacco and cannabis smoking cessation can lead to intoxication with clozapine or olanzapine. Int Clin Psychopharmacol 2002; 17(3): 141-143.

12. Tveito M, Smith RL, Hoiseth G, Molden E. The Effect of Valproic Acid on Olanzapine Serum Concentration: A Study Including 2791 Patients Treated With Olanzapine Tablets or Long-Acting Injections. J Clin Psychopharmacol 2019; 39(6): 561-566.
13. World Medical Association. Ethical principles for medical research involving human subjects [J]. Declaration of Helsinki, 2004.

14. Vicente FB, Vespa G, Miller A, Haymond $S$. Quantification of Arginine and Its Methylated Derivatives in Plasma by High-Performance Liquid Chromatography Tandem Mass Spectrometry (LC-MS/MS). Methods Mol Biol 2016; 1378: 21-30.

15. Zang YN, Dong F, Li AN, Wang CY, Guo GX, Wang Q, Zhang YF, Zhang L, de Leon J, Ruan CJ. The Impact of Smoking, Sex, Infection, and Comedication Administration on Oral Olanzapine: A Population Pharmacokinetic Model in Chinese Psychiatric Patients. Eur J Drug Metab Pharmacokinet 2021; 46(3): 353-371.

16. Leppik IE, Hovinga CA. Extended-release antiepileptic drugs: a comparison of pharmacokinetic parameters relative to original immediate-release formulations. Epilepsia 2013; 54(1): 28-35.

17. Czerwensky F, Leucht S, Steimer W. CYP1A2*1D and ${ }^{*} 1 \mathrm{~F}$ polymorphisms have a significant impact on olanzapine serum concentrations. Ther Drug Monit 2015; 37(2): 152-160.

18. Castberg I, Westin AA, Skogvoll E, Spigset O. Effects of age and gender on the serum levels of clozapine, olanzapine, risperidone, and quetiapine. Acta Psychiatr Scand 2017; 136(5): 455-464.

19. Lobo ED, Robertson-Plouch C, Quinlan T, Hong Q, Bergstrom RF. Oral olanzapine disposition in adolescents with schizophrenia or bipolar I disorder: a population pharmacokinetic model. Paediatr Drugs 2010; 12(3): 201-211.

20. Patel MX, Bowskill S, Couchman L, Lay V, Taylor D, Spencer EP, Flanagan RJ. Plasma olanzapine in relation to prescribed dose and other factors: data from a therapeutic drug monitoring service, 1999-2009. J Clin Psychopharmacol 2011; 31(4): 411-417.

21. Li A, Ji S, Yue W, Yan H, Dong F, Ruan C, Li W, Lu W, Zhang $D$, Wang C. Development of a population pharmacokinetic model of olanzapine for Chinese health volunteers and patients with schizophrenia. Bmj Open 2018; 8(8): e20070.

22. Tsuboi $T$, Bies RR, Suzuki T, Takeuchi H, Nakajima S, Graff-Guerrero A, Mamo DC, Caravaggio F, Plitman E, Mimura M, Pollock BG, Uchida H. Predicting Plasma Olanzapine Concentration Following a Change in Dosage: A Population Pharmacokinetic Study. Pharmacopsychiatry 2015; 48(7): 286-291.

Trop J Pharm Res, November 2021; 20(11): 2441 